\title{
Multilayer Miniaturized Wideband Electromagnetic Band-Gaps For Wideband Full-Duplex Transceiver
}

\author{
Marc Le Roy ${ }^{1}$, Petros Bantavis ${ }^{* 2}$, André Pérennec, Raafat Lababidi, Denis Le jeune \\ Lab-STICC, UMR CNRS 6285, \\ Université de Brest (UBO)-Ensta Bretagne, France \\ *corresponding author \\ 1'Marc.LeRoy@univ-brest.fr, ${ }^{2}$ pbadav@gmail.com,
}

\begin{abstract}
This work introduces a new design approach of multilayer Electromagnetic Band Gaps (EBGs) for wideband antenna isolation in a Full-Duplex transceiver. The design methodology is applied to a conventional type of EBGs in order to improve its initial performance in terms of bandwidth and size. By stacking multiple dielectric layers with integrated vias, an enhancement of the relative bandwidth of the band gap and a strong miniaturization of the unit cell is achieved. The dispersion diagrams of the proposed EBGs are studied and full-wave electromagnetic simulations are realized to validate the isolation level and the proposed lumped model of the final structures.

Keywords - Full-Duplex, wideband, Self-Interference cancellation, multi-layer, Electromagnetic Band Gaps.
\end{abstract}

\section{INTRODUCTION}

Full-Duplex (FD) technology has recently appealed the attention in the wireless communication networks and promises to double the throughput and provide better spectrum flexibility [1]. A FD transceiver can theoretically enhance the Signal to Noise Ratio (SNR) on its own receiver and double the spectral efficiency by transmitting and receiving simultaneously on the same frequency channel. The main challenge in a FD radio is to cancel the Self-Interference (SI) signal that leaks from the transmitter to the receiver and degrades the SNR. Recent works in [2]-[3] achieve high level of SI-signal cancellation but feature narrowband performance. In a FD transceiver such as the one depicted in Fig. 1, the main sources of self-interference come from near-field or freespace coupling and also from an indirect surface-wave leakage between antennas as well. This latter coupling appears when the two antennas share the same substrate or the same ground plane. Moreover, impedance mismatch, transmitter and receiver impairments, non-linearity in the RF front end components and multiple reflections of the emitted signal (Non-Line of Sight (NLOS) transmission from the Tx to Rx) contribute to some additional but lower SI level into the receiver. Thus, for an efficient FD transceiver system, the cancellation techniques need to be considered gradually starting from the antenna section to the digital one, passing through the RF/analog part.

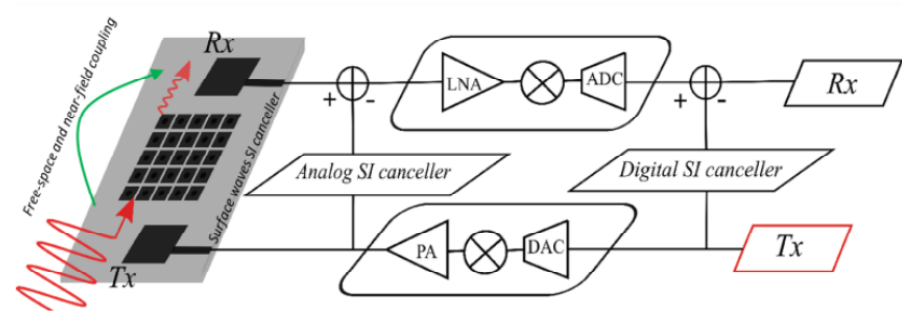

Fig. 1. Full-Duplex transceiver with the proposed EBGs to contribute as antenna self-interference (SI) canceller.

The context of this work relies on the design of broadband SI cancellation at the antenna level in a wideband FD transceiver. The major SI contributions, i.e. the near-field or free space mutual couplings, can be respectively reduced by reasonably increasing the distance between antennas (more than about $\lambda /(2 \pi)$ ) and/or by designing TX antennas with radiation patterns that exhibit minimum in directions toward RX antenna. Once these antenna-level SI signal components are strongly reduced, surface waves, which are guided here along the interface between the ground plane and the dielectric/air interface, become the most important SI contribution. Then, suppressing surface-wave coupling is of prime interest in FD systems where SI signal cancellation level at the antenna/analog section are expected to be better than -40 to $-60 \mathrm{~dB}$ depending on the foreseen applications. EBGs with vias have been widely studied and are considered as the best candidates to reduce the mutual coupling due to surfaces waves [4]. However, conventional EBGs present narrowband performance and suffer from large sizes. In this paper, a multilayer stacked EBG is studied and by increasing the number of dielectric layers, the size of the unit cell is drastically minimized. Moreover, as the size of the unit cell is reduced, the relative bandwidth of the bandgap is increased while a second bandgap is produced at higher frequencies. The present multilayer technique is very promising as it can be applied to different types of EBGs and creates wide frequency bandgaps suitable for any application that demand to reduce mutual coupling due to surface waves in a multilayer stacked antennas configuration. 


\section{WIDEBAND MULTILAYER EBG}

In low-profile planar antennas, such as microstrip antenna array, surface wave modes are excited into the dielectric substrate. In that grounded dielectric slab, several surface wave modes [5] are supported depending on the thickness $h$ and permittivity $\varepsilon_{r}$ of the substrate: $\mathrm{TM}_{0}$ is the dominant mode and propagates at all frequencies and $\mathrm{TE}_{\mathrm{n}}$ and $\mathrm{TM}_{\mathrm{n}}$ waves above their respective cut-off frequencies.

For TM modes:

$$
f_{c}=\frac{n c}{2 h \sqrt{\varepsilon_{r}-1}}, \quad n=0,1,2, \ldots
$$

For TE modes:

$$
f_{c}=\frac{(2 n-1) c}{4 h \sqrt{\varepsilon_{r}-1}}, \quad n=1,2,3, \ldots
$$

where $c$ is the free-space light-velocity. In relation with (1)-(2), the substrate thickness can be chosen in a manner that the cutoff frequency of the lowest TE mode is shifted above the application operating frequency band. However, $\mathrm{TM}_{0}$ mode will always be excited at the edge of the planar antenna. In order to thwart surface waves in a grounded-dielectric slab, EBG with vias are required. In classical mushroom singledielectric-layer EBG such as the one depicted in Fig. 2, an effective capacitance $\mathrm{C}$ is used to modelize the existing electric fields in the gap of dimension $g$ between the square metallic patches. Whereas, an inductance represents the inductive path of the current flow from one patch to the adjacent one (patch-via-ground-via-patch, see Fig. 2), and thus depends on the substrate thickness and permeability. In mushroom-type EBGs, the surface-wave frequency bandgap is defined by its centre angular frequency $\omega_{0}(3)$, its bandwidth $B W(4)$, and the impedance surface $Z$ (5) which can be roughly predicted by this lumped model [6].

$$
\begin{aligned}
& \omega_{0}=\frac{1}{\sqrt{L C}} \\
& B W=\frac{\Delta \omega}{\omega_{0}}=\frac{\sqrt{L / C}}{\sqrt{\mu_{0} / \varepsilon_{0}}} \\
& Z=j \frac{\omega L}{1-\omega^{2} L C}
\end{aligned}
$$

Mushroom-like synthesis equations in function of the geometry and cell arrangement can be found in [6], and a square lattice was chosen due to its higher bandwidth [6].

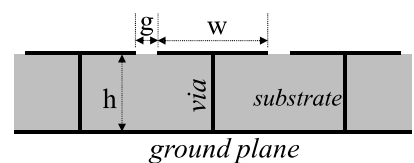

a)

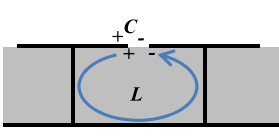

b)

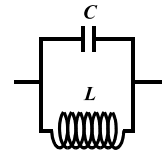

c)
Fig. 2. Side view of a classical single layer EBG with square patterns (a); unit cell behaviour (b); and equivalent lumped model (c).

It has been shown that utilizing multiple layers in any type of EBG can be used to minimize the size of a unit cell. For example, in [7], the capacitance is increased by overlapping plates which indeed brings lower resonance frequency but also mitigates the relative bandwidth as evidenced by (4). The multilayer EBG structure of [8]-[9] simultaneously allows enlarging capacitance and inductance; however, the structure proposed in [9] is unidimensional, whereas the interleaved multilayer EBG of [8] requires blind via holes. Moreover, they are dedicated to reduce noise-switching between power plane and ground in high-speed digital circuits. In this paper, a multilayer EBG structure that increases both the inductance and capacitance values is proposed in order to simultaneously miniaturize the unit-cell size and to keep a wide surface-wave stopband bandwidth. Moreover, a dual-band behaviour is achieved. An equivalent lumped model is proposed and validated through electromagnetic simulations. The EBG stack proposed in this paper is dedicated to decrease the coupling level between planar antennas, but the same approach can be applied to reduce switching noise in mixed analog/digital high-speed circuit.

\section{A. Multilayer EBG lumped model}

The present design methodology is based on stacking multiple dielectric layers with integrated through via holes between copper layers that include square patches excepted for the full metallic ground plane at the bottom.

Globally, stacking multiple dielectric layers with integrated vias increases the total capacitance of unit cell as well as the total inductance. However, even by only considering the major contributions, i.e. via inductances and gap capacitances between patches as depicted in Fig. 3.a, it's obvious that the total inductance and the total capacitance of a stacked unit cell do not just correspond to the sum of each contribution independently.

In this paper, a new equivalent lumped model is proposed. In order to simplify the calculation, the capacitances $\mathrm{C}$ between adjacent mushrooms are considered equal regardless the layer. Moreover, the whole inductance $L_{T}$ of each single layer is subdivided into three parts, corresponding to the via contribution, $L_{V}$, and to the ground one, $L_{G}$. In this case, the multilayer lumped equivalent model of the impedance surface can be simplified by a single equivalent layer configuration that takes into account the upper layer and the corresponding equivalent model of the lower layers down to the ground plane as depicted in Fig. 3a. Starting from the single layer impedance $Z_{1}$, the 2-layer stack impedance $Z_{2}$ can be deduced and so on. For example, the surface impedance $Z_{\mathrm{n}}$ for a $\mathrm{n}$ layer-stack is expressed as:

$Z_{n}=Z_{C} \|(j 2 L_{V} \omega+\underbrace{\left(Z_{C} \|\left(j 2 L_{V} \omega+\left(Z_{C} \|\left(\ldots . .+Z_{1}\right)\right)\right)\right)}_{Z_{n-1}})$

Where $Z_{C}=1 /(j C \omega)$. So, the impedance for a given number of layers can be easily computed. In the next section, the relevance of this staked EBG structure is put forward and the proposed lumped-model is validated by computing the corresponding dispersion diagrams and full-wave electromagnetic (EM) simulations. 


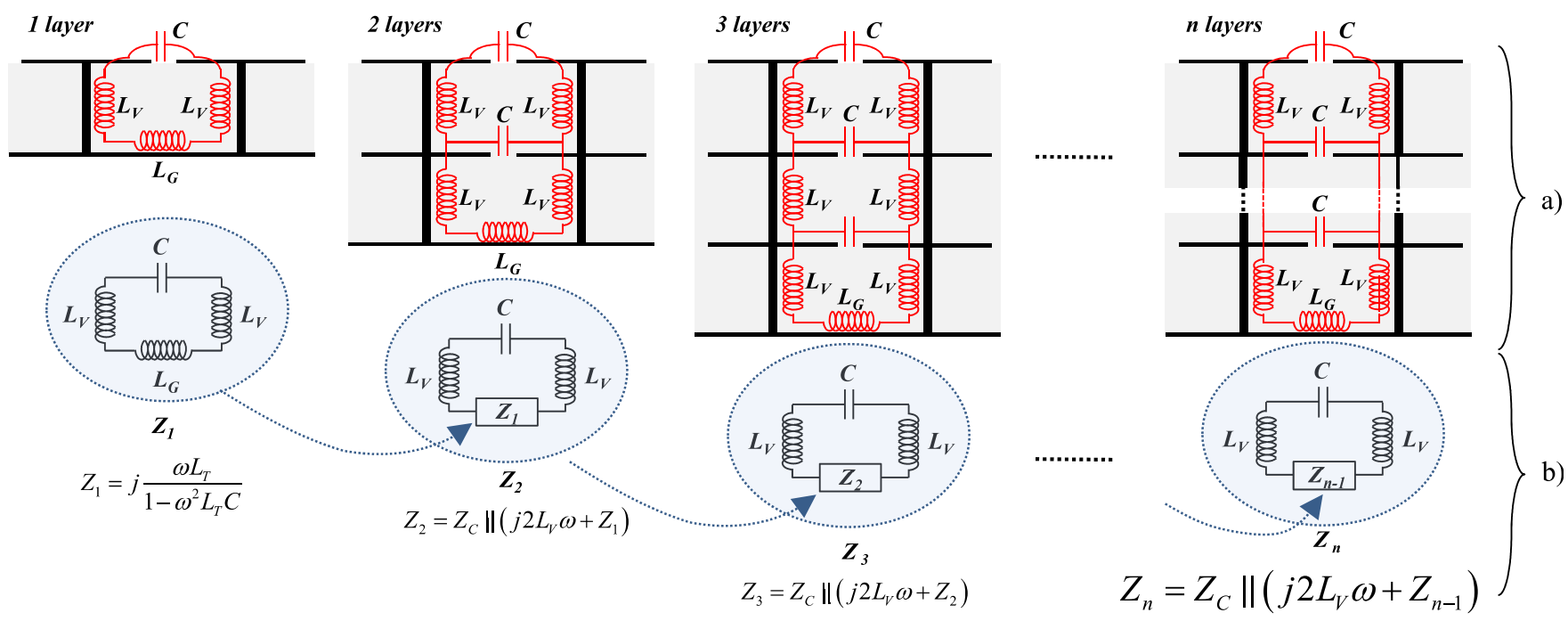

Fig. 3. Illustration of the major contributions between adjacent cells for different number of stacked layers (a); equivalent lumped model and corresponding surface impedance expressions (b).

\section{B. Model validation and dispersion diagram evaluation}

In Fig. 4a, the dispersion diagrams are obtained for the unit cells of Fig. 3a for a stack of 1, 3, 5 and 10 layers by using eigenmode solver of CST Microwave studio. The substrate layers have all a dielectric permittivity $\varepsilon_{r}$ of 2.2 and a thickness $h$ of $1.6 \mathrm{~mm}$. The gap, $g$, between adjacent mushrooms is of $0.3 \mathrm{~mm}$ and the metallization thickness is $35 \mu \mathrm{m}$. As shown in Fig. $4 \mathrm{a}$, the single-layer EBG produces a first bandgap between 11-20 GHz with a relative bandwidth of $58 \%$. After stacking three layers, the first band gap is created between $5-10 \mathrm{GHz}$ with a relative bandwidth slightly increased to $66 \%$. Finally, as the bandgap is shifted to lower frequencies with the same unit-cell dimensions, multi-layer EBG (M-EBG) stacking can be considered as a way to minimize the relative lateral dimension of the unit cell. Additionally, a second band gap appears in the $12-14 \mathrm{GHz}$ band. In Fig. 4b, when introducing 5 layers, the first band gap is produced at a lower frequency band (3.3-6.5 GHz) and the second one is located at 9-10.5 GHz. Thus, as expected, adding multiple stacked layers allows shifting down the resonance frequency for both bands while slightly increasing the relative bandwidth for the first one.

In Table I, the results extracted from the dispersion diagrams for the first and second bandgaps are presented for the structure of Fig. 3a from 1 to 10 stacked dielectric layers with through via holes. To go further in the validation of the proposed design performance, full-wave EM simulations were run to evaluate the isolation of a finite $5 \times 5$ structure with the proposed multilayer stacked EBGs. A coplanar line was designed to excite the structure and also the TE and TM surface waves in the dielectric substrates over a wider bandwidth than with traditional patch antennas. Indeed, validating the wideband multilayer EBG would require designing wideband and multiband multilayer planar antennas that cover the corresponding bandgaps for each number of stacked layers. Designing on the same stacked structure wideband planar RX/TX antennas and their EBG isolation circuit is our future work toward the implementation of an antenna section with improved SI cancellation for FD frontend. Fig. 5 shows the insertion loss $\left(\mathrm{S}_{21}\right)$ of the finite $5 \times 5$ structure using the proposed unit cell of Fig. 3a with 1, 3, 5 and 10 stacked layers (the inset presents the finite $5 \times 5$ structure with 5 layers). This figure confirms the resonance frequency and the bandwidths of the bandgaps, the dual-band performance of all the multilayer EBGs and also the high isolation level in the band gaps. If $\lambda_{5 \mathrm{GHz}}(60 \mathrm{~mm})$ is the free space wavelength at $5 \mathrm{GHz}$, the EBG pattern periodicity is of $0.04 \lambda_{5 \mathrm{GHz}}(2.4 \mathrm{~mm})$, and $-45 \mathrm{~dB}$ wideband isolation can be obtained between antennas operating at that frequency and which are longitudinally spaced from only $0.2 \lambda_{5 \mathrm{GHz}}$.

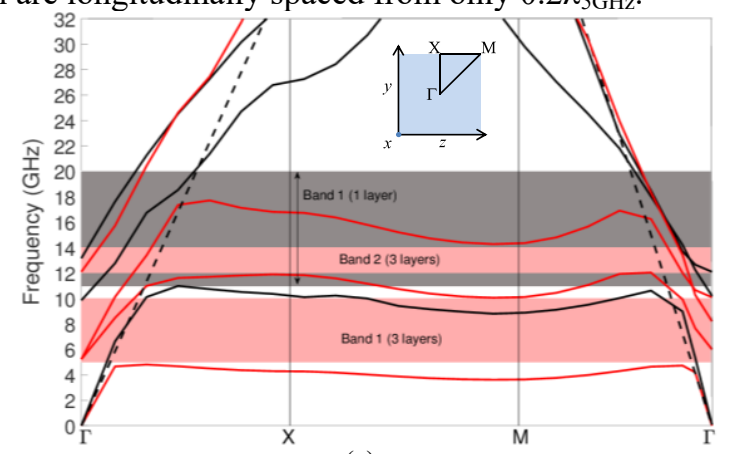

(a)

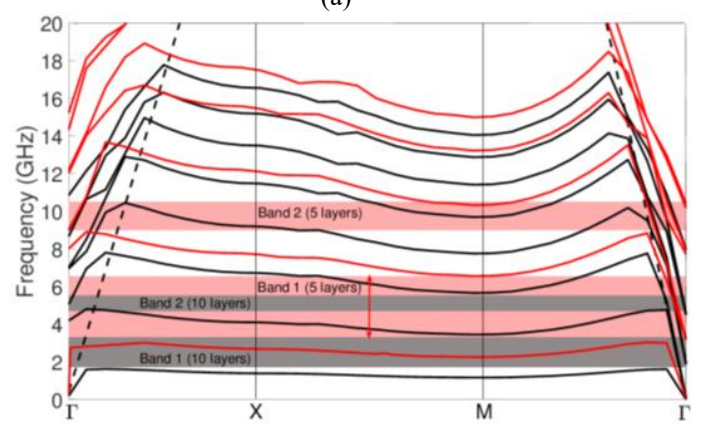

(b)

Fig. 4. (a) Dispersion diagrams of the Multilayer EBG (M-EBG) of Fig. 3a for 1 and 3 stacked layers; and (b) for 5 and 10 stacked layers. Dimensions: $\mathrm{w}=2.25 \mathrm{~mm}, \mathrm{~g}=0.3 \mathrm{~mm}, \mathrm{~h}=1.6 \mathrm{~mm}$. 
Table 1. Bandwidths $(B W)$ and bandgaps for 1 to 10 layers of M-EBG of Fig. 3a.

\begin{tabular}{|c|c|c|c|c|c|c|c|c|c|c|}
\hline Number of layers & 1 & 2 & 3 & 4 & 5 & 6 & 7 & 8 & 9 & 10 \\
\hline $1^{\text {st }}$ Bandgap (GHz) & $11-20$ & $6.7-13.2$ & $5-10$ & $3.6-8$ & $3.3-6.5$ & $2.5-6.4$ & $2.4-4.9$ & $2.1-4.5$ & $2-4.5$ & $1.8-3.8$ \\
\hline $2^{\text {nd }}$ Bandgap $(\mathrm{GHz})$ & - & - & $12-14$ & $10-12.2$ & $9-10.5$ & $8.1-9.5$ & $7.5-9.6$ & $7.1-8.2$ & $6.5-7.2$ & $5-5.7$ \\
\hline$B W(\%)$ & 58 & 65.3 & 66.6 & 75.8 & 65 & 67.4 & 68.4 & 72.7 & 76.9 & 71.4 \\
\hline
\end{tabular}

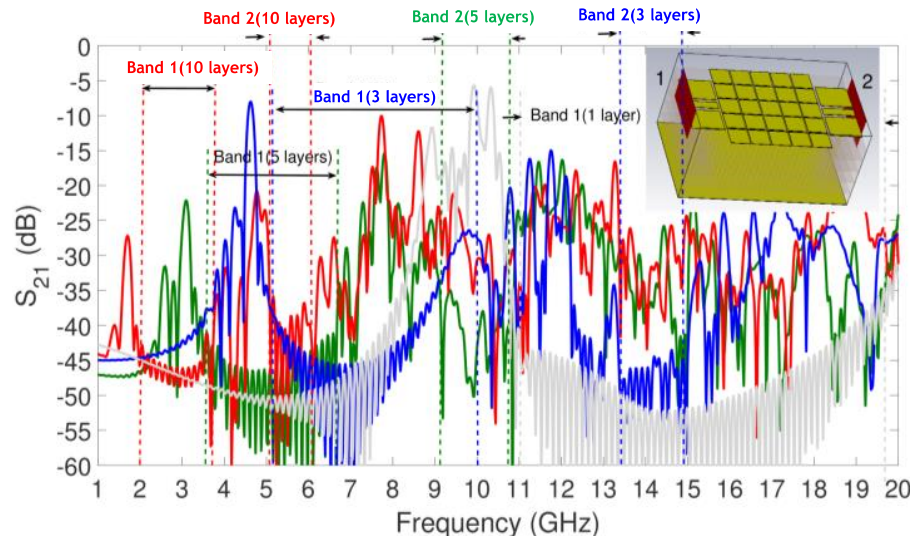

Fig. 5. Insertion loss of the multi-layer EBG for 1, 3, 5 and 10 layers.

The previous simulations are used as a reference to validate the proposed lumped model. First, the edgecapacitance value $C$ for the gap between adjacent mushrooms of the top layer can be calculated from the following equation which is obtained from classical conformal mapping [6]:

$$
C=\frac{w \varepsilon_{0}\left(\varepsilon_{r 1}+\varepsilon_{r 2}\right)}{\pi} \cosh ^{-1}\left(\frac{w+g}{w}\right)
$$

For the top layer, $\varepsilon_{r 1}=1$ and $\varepsilon_{r 2}=2.2$ and for internal layers $\varepsilon_{\mathrm{r} 2}=\varepsilon_{r 1}=2.2$. The global inductance value $L_{T}$ for the single layer EBG is obtained from (7).

$$
L_{T}=\mu_{0} h
$$

Then, using classical via inductance formula, the via contribution, $L_{V}$, is calculated for a $200 \mu \mathrm{m}$ diameter and thus the ground one $L_{G}$ is deduced. For our example, we get $L_{T}=2 \mathrm{nH}, \mathrm{L}_{\mathrm{V}}=660 \mathrm{pH}, L_{G}=0.69 \mathrm{pH}$, and $C=56 \mathrm{fF}$ for the top layer. Fig. 6 shows a comparison between the first bandgap centre frequencies obtained from the lumped model and the EM simulations in function of the number of layers.

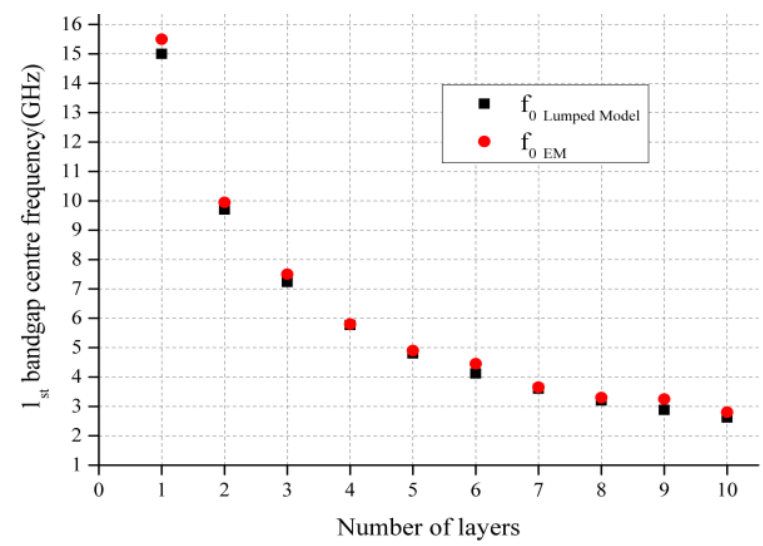

Fig. 6. Comparison of the centre bandgap frequencies obtained from the lumped model and from EM simulations.
As for classical-EBG lumped LC-model, this approach provides relations to estimate an approximate resonance frequency and the surface impedance in an initial design synthesis. Moreover, this multi-layer stack model is only valid for the lowest frequency bandgap. The proposed approach could also be applied to synthesize specific bandgap with different mushroom sizes and substrate thicknesses.

\section{CONCLUSION}

In this work, a design methodology of multilayer stacked electromagnetic band gaps is presented. It has been proved that increasing the number of stacked dielectric layers with through via holes allows to: i) minimize the unit-cell size by shifting toward low frequency the obtained stopbands, ii) achieve dual-band performance and iii) enhance the relative bandwidth of the first band gap. An equivalent simplified lumped model was also proposed and validated by dispersion diagrams and EM simulations. Here, this stacked EBG structure serves as a complementary technique to improve SI signal cancellation in wideband Full-Duplex RF front-end by suppressing surface waves. This low-complexity approach doesn't require blind via hole and can also be easily applied to minimize mutual coupling in MIMO antenna systems or to improve wideband noise-suppression in high-speed digital circuits. In the overall project, this technique is planned to be used in conjunction with broadband stacked planar antennas where, moreover, internal EBG layers could serve as highimpedance ground for these latter.

\section{REFERENCES}

[1] D. Bharadia, E. McMilin, and S. Katti, "Full duplex radios," SIGCOMM Comput. Commun. Rev., vol. 43, no. 4, pp. 375-386, 2013.

[2] M. Jain, J. I. Choi, T. Kim, D. Bharadia, S. Seth, K. Srinivasan, P. Levis, S. Katti, and P. Sinha, "Practical, real-time, full duplex wireless," 17th Annual International Conference on Mobile Computing and Networking, ser. MobiCom 11, New York, 2011, pp. 301-312.

[3] B. Debaillie, D. J. van den Broek, C. Lavin, B. van Liempd, E. A. M. Klumperink, C. Palacios, J. Craninckx, and B. Nauta, "RF selfinterference reduction techniques for compact full duplex radios," IEEE 81st Vehicular Technology Conference, May 2015, pp. 1-6.

[4] F. Yang and Y. Rahmat-Samii, "Microstrip antennas integrated with electromagnetic band-gap (ebg) structures: a low mutual coupling design for array applications," IEEE Transactions on Antennas and Propagation, vol. 51, no. 10, pp. 2936-2946, Oct 2003.

[5] D. M. Pozar, Microwave engineering, John Wiley \& Sons, 2009.

[6] C. A. Balanis, Advanced engineering electromagnetics, 2nd ed. Hoboken, N.J: John Wiley \& Sons, 2012.

[7] T. Kamgaing and O. M. Ramahi, "Multiband electromagnetic-bandgap structures for applications in small form-factor multichip module packages," IEEE Trans. MTT, vol. 56, no. 10, pp. 2293-2300, Oct 2008.

[8] Y. Kim, M. Kim, and Y. Yang, "Compact electromagnetic band gap structure for wideband noise suppression in multilayer pcb," $A P M C$, vol. 2, Dec 2015, pp. 1-3.

[9] L. Peng, C. L. Ruan, and Z. Q. Li, "A novel compact and polarization dependent mushroom-type ebg using csrr for dual/triple-band applications," IEEE MWCL, vol. 20, no. 9, pp. 489-491, Sept 2010. 